\title{
Temporomandibular articular disorders can be alleviated with surgery
}

\author{
Which surgical procedures, if any, can effectively treat temporomandibular \\ articular disorders?
}

\section{Reston JT, Turkelson CM. Meta-analysis of surgical treatments for temporomandibular articular disorders. J Oral Maxillofac Surg 2003; 61:3-10}

Data sources Medline, Embase and a variety of other sources were searched for English-language articles. Non-journal publications, conferences proceedings from professional organisations and from private and government agencies were screened. Bibliographies and reference lists from peer-reviewed and 'grey' literature were also reviewed.

Study selection To meet the inclusion criteria articles must have reported studies of at least 10 patients, involve diagnoses of disc displacement with reduction (DDwR) or without reduction (DDw/oR) or degenerative joint disease (DJD) and include at least one of the following surgical techniques: arthrocentesis; arthroscopy; discectomy without replacement or disc repair/repositioning.

Data extraction and synthesis The outcome measured was the proportion of patients who reported improvement after treatment. Study success rates were recalculated on an intent-to-treat basis. Metaanalyses were performed along with meta-regressions where heterogeneity was an issue. Three classes of meta-analyses were performed using different artificial, untreated, control groups assuming three levels of spontaneous improvement, namely $0,37.5$ and $75 \%$.

Results Twenty-two studies, comprising 30 patient groups and sample sizes of 11-237 patients, met the inclusion criteria. For studies of patients with DDwR, at the 0 and $37.5 \%$ rates of control group improvement, arthroscopy and disc repair/repositioning resulted in treatment effects significantly greater than zero, with no significant differences in the efficacy of these two surgical techniques. For studies of patients with DDw/OR, the proportion of patients who improved after arthroscopy or arthrocentesis was significantly greater than zero at all three levels of estimated control improvement. Disc repair effect size was not significant at the $75 \%$ rate.

Conclusions Surgical treatments appear to have some efficacy for people who have temporomandibular articular disorders that do not respond to nonsurgical therapies.

\begin{abstract}
Appraisal
Objectives The present paper is a reappraisal of the data used by Reston and Turkelson in the first available published meta-analysis of temporomandibular joint surgery.

Study design A systematic review of the data, as used by the authors, was critically evaluated, using additional inclusion criteria. Results Of all the citations reviewed, 23 out of 30 cited studies were found to meet the current inclusion criteria.

Conclusion Although the available literature consists of retrospective and prospective case series and two randomised control trials (RCT). It appears that the newer techniques of arthrocentesis and arthroscopy are associated with higher success rates than the techniques most common in the past, such as discectomy and disc repair.
\end{abstract}

Address for correspondence: Dr CM Turkelson, ECRI, 5200 Butler Pike, Plymouth Meeting PA 19462-1298, USA. E-mail: cturkelson@ecri.org

\section{Introduction}

Temporomandibular disorder causes pain and dysfunction in many patients world-wide. A small proportion of the patients do not improve following conservative therapies. ${ }^{1}$ Numerous open joint procedures were developed to reposition and reshape the displaced disc or deformed disc. Today, these modalities range from a minimally invasive technique such as arthroscopy to open temporomandibular joint surgery. Arthroscopic surgery is believed to be less invasive than open temporomandibular joint surgery. It is unclear to what degree arthroscopy is superior, however. Open temporomandibular surgery requires increased intra-operative time and greater risk to adjacent tissues or structures.

Currently, there is a lack of published prospective, randomised studies with long-term results to assist the surgeon in choosing a particular option, and therefore the personal experience of the surgeon may become the determining factor controlled trials as pointed out by Reston and Turkelson in the paper above.

\section{Methods}

We devised similar methodological criteria for analysing the same articles as those selected in the above paper. We used the following section criteria:

(1) At least 10 patients must have enrolled in the study.

(2) Patients included had DDw/oR.

(3) Articles should report on the different treatments: arthrocentesis, arthroscopy, discectomy and disc repair.

The other criteria for inclusion were:

(4) Follow-up duration of 12 months or longer.

(5) Improvement is based on measurable increase in maximal mouth-opening.

\section{Results}

Of the papers that were highlighted, four articles were excluded because of short time periods or included patients with DDw/oR. For the purpose of analysis, several discrete treatment types were identified in the 23 selected articles. These included:

Arthrocentesis. This is the simplest and least invasive of all the surgical techniques. It involves the placement of two needles in the superior joint space for lavage and instillation of corticosteroid or sodium hyaluronate to treat internal derangement.

Arthroscopy. This is the placement of an arthroscope with an attached camera into the superior space for examination, lavage, lysis, and arthroplasty and disc stabilisation.

Disc repair is the reposition of the articular disc to a more normal anatomical relation with the condyle and the fossa is usually undertaken when the disc is displaced but free of disease or structural deformity.

Discectomy is the complete removal of the disc. It was one of the first intra-articular temporomandibular-joint surgical procedures described. Discectomy is undertaken when the disc is found to be diseased or structurally compromised as a result of tears, perforations or persisting symptoms of pain and dysfunction after surgery.

The individual papers, with treatment, type of study, sample number and improvement, are listed in Table $1 .$. The results 
Table 1. Summary of studies used in the meta-analysis.

\begin{tabular}{|c|c|c|c|c|c|c|}
\hline Author & Type & No. patients & Treatment & Follow-up & Patients showing improvement & Improvement (\%) \\
\hline Nitzan et $a P^{5}$ & $P$ & 39 & Arthrocentesis & 16.6 & 38 & 0.974 \\
\hline Dimitroulis et $a l^{6}$ & $P$ & 46 & Arthrocentesis & 21 & 45 & 0.978 \\
\hline Nitzan et $a l^{7}$ & $P$ & 17 & Arthrocentesis & 14 & 16 & 0.941 \\
\hline Miyamoto et $a l^{8}$ & RCT & 35 & Arthroscopy & 12 & 34 & 0.971 \\
\hline Miyamoto et a 9 & $\mathrm{RCT}$ & 63 & Arthroscopy & 34.4 & 57 & 0.904 \\
\hline Kurita et $a l^{10}$ & $P$ & 14 & Arthroscopy & 28.5 & 12 & 0.857 \\
\hline Moore et $a l^{11}$ & P & 63 & Arthroscopy & 48 & 55 & 0.873 \\
\hline Zeitler et $a l^{12}$ & $\mathrm{R}$ & 23 & Arthroscopy & 13 & 20 & 0.869 \\
\hline Clark et $a l^{13}$ & $\mathrm{R}$ & 18 & Arthroscopy & 30 & 16 & 0.888 \\
\hline Indresano et al ${ }^{14}$ & $\mathrm{R}$ & 33 & Arthroscopy & 18.9 & 27 & 0.818 \\
\hline Fridrich et $a l^{3}$ & $\mathrm{RCT}$ & 11 & Arthroscopy & 12.9 & 9 & 0.818 \\
\hline Trumpy et $a l^{15}$ & $\mathrm{R}$ & 13 & Disc repair & 60 & 10 & 0.769 \\
\hline Kuwahara et al ${ }^{15}$ & $\mathrm{R}$ & 90 & Disc repair & 12 & 83 & 0.922 \\
\hline Hall et $a l^{16}$ & $\mathrm{R}$ & 20 & Disc repair & 18.1 & 13 & 0.650 \\
\hline Zeitler et $a l^{12}$ & $\mathrm{R}$ & 24 & Disc repair & 25.2 & 20 & 0.833 \\
\hline Trumpy et $a l^{15}$ & $\mathrm{R}$ & 17 & Discectomy & 60 & 16 & 0.941 \\
\hline Holmlund et $a l^{17}$ & $P$ & 72 & Discectomy & 12 & 60 & 0.833 \\
\hline Eriksson et $a l^{18}$ & $\mathrm{R}$ & 21 & Discectomy & 60 & 18 & 0.857 \\
\hline Brown et $a l^{19}$ & $\mathrm{R}$ & 214 & Discectomy & 180 & 182 & 0.850 \\
\hline Takaku et al ${ }^{20}$ & $\mathrm{R}$ & 39 & Discectomy & 111.6 & 37 & 0.948 \\
\hline Kuwahara et al 21 & $\mathrm{R}$ & 74 & Discectomy & 12 & 70 & 0.945 \\
\hline
\end{tabular}

$\mathrm{P}$, prospective study; $\mathrm{R}$, retrospective study.

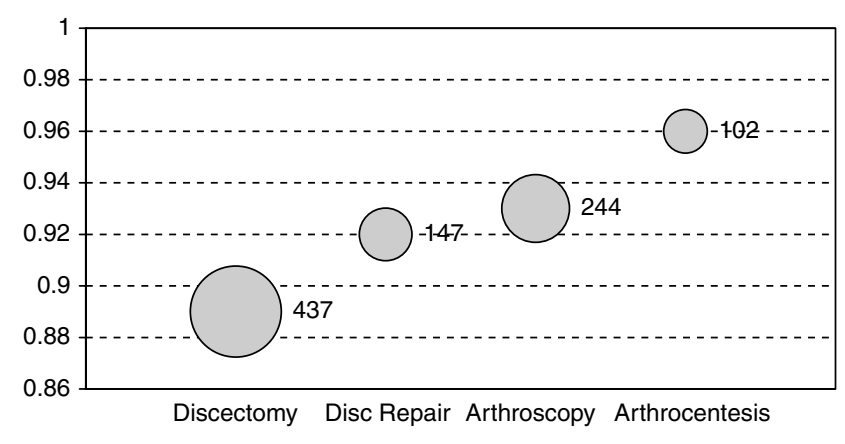

\begin{tabular}{lcc} 
Data Table & & \\
\hline & Patients & Proportion (\%) \\
\hline Arthrocentesis & 102 & 0.964 \\
Arthroscopy & 244 & 0.938 \\
Disc repair & 181 & 0.922 \\
Discectomy & 437 & 0.896
\end{tabular}

Figure 1. Summary of success by treatment type.

indicate that arthrocentesis gives the greatest degree of improvement after intervention, compared with the historical control of discectomy and disc repair. The comparison of arthroscopy and arthrocentesis, however, showed that enrolled numbers in trials for arthroscopy were greater (Figure 1).

\section{Discussion}

The purpose of this investigation was to systematically evaluate the treatments that have been described for the treatment of the temporomandibular joint with DDw/oR. Unfortunately, the substantial degree of variability within individual studies precludes a quantitative analysis of the data. Applying a uniform quantitative analysis to control variables and unify data could be misleading.

Regardless of the differences in patient population, some useful analysis can be carried out by applying systemic review methods to the studies. A total of 23 papers provided enough data to allow an assessment of the effect of treatment on success rate. We acknowledge that these studies are not equal in value, however. For the treatments described, the one most likely to be successful for DDw/ oR appears to be arthrocentesis, with the next most successful being arthroscopy.

Arthrocentesis has emerged as an excellent treatment in people who have recent onset of painful limitation of mandibular opening. Nitzan $e t a l^{2}$ were the first to have reported on clinical outcomes in 39 patients who underwent arthrocentesis of 40 joints. Other studies have specifically compared the results of arthrocentesis and arthroscopy. Fridrich et $a^{3}$ performed a prospective study in 19 patients with internal derangement unresponsive to nonsurgical therapy randomised into arthroscopy and arthrocentesis groups. The authors reported $82 \%$ success for the arthroscopy group and $75 \%$ with the arthrocentesis group, based on improvement in maximum incisal opening and subjective pain scores. This was deemed not significant different by the authors. Another investigator, Murakami ${ }^{4}$ compared the efficacy of nonsurgical therapy, arthrocentesis and arthroscopy in 108 patients with internal derangement and closed lock. Arthrocentesis had a success rate of $70 \%$ whereas for arthroscopy this was $91 \%$, the author again concluding that the difference was not significant.

\section{Conclusion}

Based on this analysis, we conclude that disc repair and discectomy are good historical controls. Surgical arthrocentesis and arthroscopy are effective for DDw/oR patients. Better-designed trials are needed before one can accurately determine the magnitude of the benefits of temporomandibular articular surgery.

\section{CH Ng, JB Lai, F Victor, JF Yeo}

Department of Oral and Maxillofacial Surgery, National University of Singapore, Singapore

1. Santacattrina A, Paoli M, Peretta, Bambace A, Belerame A. A comparison between horizontal splint and repositioning splint in the treatment of 'disc dislocation with reduction'. Literature meta-analysis. J Oral Rehab 1998; 25:81-88.

2. Nitzan DW, Dolwick MF, Heft MW. Arthroscopic lavage and lysis of the temporomandibular joint: a change in perspective. J Oral Maxillofac Surg 1990; 48:498. 
3. Fridrich $\mathrm{KL}$, Wise JM, Zeitler DL. Prospective comparison of arthroscopy and arthrocentesis for temporomandibular joint disorders. J Oral Maxillofac Surg 1996; 54:816-820.

4. Murakami KI, Matsuki M, Lizuka T. Recapturing the persistent anterior displaced disc by mandibular manipulation after pumping and hydraulic pressure to the upper joint cavity of the temporomandibular joint. J Craniomandib Pract 1987; 5:18.

5. Nitzan DW, Samson B, Better H. Long-term outcome of arthrocentesis for sudden onset, persistent, severe closed lock of the temporomandibular joint. J Oral Maxillofac Surg 1997; 55:151-157.

6. Dimitroulis G, Dolwick MF, Martinez A. Temporomandibular joint arthrocentesis and lavage for the treatment of closed lock: a follow-up study. $\mathrm{Br}$ J Oral Maxillofac Surg $1995 ; 33: 23-26$.

7. Nitzan DW, Dolwick MF, Martinez GA. Temporomandibular joint arthrocentesis: a simplified treatment for severe, limited mouth opening. J Oral Maxillofac Surg 1991; 49:1163-1167.

8. Miyamoto $\mathrm{H}$, Sakashita $\mathrm{H}$, Miyata M. Arthroscopic surgery of the temporomandibular joint: comparison of two successful techniques. $\mathrm{Br}$ J Oral Maxillofac Surg 1999; 37:397-400.

9. Miyamoto H, Sakashita H, Miyata M. Arthroscopic management of temporomandibular closed lock. Aust Dent J 1998; 43:301-304.

10. Kurita K, Goss AN, Ogi N. Correlation between preoperative mouth opening and surgical outcome after arthroscopic lysis and lavage in patients with disc displacement without reduction. J Oral Maxillofac Surg 1998; 56 : 1394-1397.

11. Moore LJ. Arthroscopic surgery for the treatment of restrictive temporomandibular joint disease. A retrospective longitudinal study. In Advances in Diagnostic and Surgical Arthroscopy of the Temporomandibular Joint Edited by Clark G, Sander B, Bertolami C. Philadelphia: Saunders; 1993; 35-40.

12. Zeitler D, Porter BA. A retrospective study comparing arthroscopic surgery with arthrotomy and disc repositioning. In Advances in Diagnostic and Surgical
Arthroscopy of the Temporomandibular Joint Edited by Clark G, Sander B, Bertolami C. Philadelphia: Saunders; 1993; 47-60.

13. Clark GT, Moody DG, Saunders B. Arthroscopic treatment of temporomandibular joint locking resulting from disc derangement: two year results. J Oral Maxillofac Surg 1991; 49:157-164.

14. Indresano AT. Arthroscopic surgery of the temporomandibular joint: report of 64 patients with long-term follow-up. J Oral Maxillofac 1989; 47:439-441.

15. Trumpy IG, Lyberg T. Surgical treatment of internal derangement of the temporomandibular joint: long-term evaluation of three techniques. J Oral Maxillofac Surg 1995; 53:740-746.

16. Kuwahara T, Bessette RW, Maruyama T. A retrospective study on the clinical results of temporomandibular joint surgery. Cranio 1994; 12:179.

17. Hall MB. Meniscoplasty of the displaced temporomandibular joint meniscus without violating the inferior joint space. J Oral Maxillofac Surg 1984; 42:788.

18. Holmlund $A B$, Gynther $G$, Axelsson S. Diskectomy in treatment of the temporomandibular joint: follow-up at 1, 3 and 5 years. Oral Surg Oral Med Oral Path 1993; 76:266-271.

19. Eriksson L, Westesson PL. Temporomandibular joint discectomy. No positive effect of temporary silicone implant in a 5-year follow-up. Oral Surg Oral Med Oral Path 1992; 74:259-272.

20. Brown WA. Internal derangement of the temporomandibular joint: review of 214 patients following meniscectomy. Can J Surg 1980; 23:30-32.

21. Takaku S, Toyoda T. Long term evaluation of discectomy of the temporomandibular joint. J Oral Maxillofac Surg 1994; 52:722-726.

Evidence-Based Dentistry (2005) 6, 48-50.

doi:10.1038/sj.ebd.6400328 